\title{
Gastroesophageal Reflux Symptoms and Comorbid Asthma and Posttraumatic Stress Disorder Following the 9/11 Terrorist Attacks on World Trade Center in New York City
}

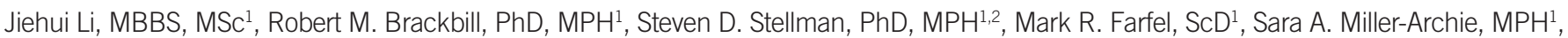
Stephen Friedman, MD, MPH${ }^{1}$, Deborah J. Walker, $\mathrm{PhD}^{1}$, Lorna E. Thorpe, $\mathrm{PhD}^{3}$ and James Cone, MD, $\mathrm{MPH}^{1}$

OBJECTIVES: $\quad$ Excess gastroesophageal reflux disease (GERD) was reported in several populations exposed to the September 112001 (9/11) terrorist attacks on the World Trade Center (WTC). We examined new onset gastroesophageal reflux symptoms (GERS) since $9 / 11$ and persisting up to 5-6 years in relation to 9/11-related exposures among the WTC Health Registry enrollees, and potential associations with comorbid asthma and posttraumatic stress disorder (PTSD).

METHODS: $\quad$ This is a retrospective analysis of 37,118 adult enrollees (i.e., rescue/recovery workers, local residents, area workers, and passersby in lower Manhattan on 9/11) who reported no pre-9/11 GERS and who participated in two Registry surveys 2-3 and 5-6 years after 9/11. Post-9/11 GERS (new onset since 9/11) reported at first survey, and persistent GERS (post-9/11 GERS reported at both surveys) were analyzed using log-binomial regression.

RESULTS: $\quad$ Cumulative incidence was $20 \%$ for post-9/11 GERS and $13 \%$ for persistent GERS. Persistent GERS occurred more often among those with comorbid PTSD (24\%), asthma (13\%), or both (36\%) compared with neither of the comorbid conditions (8\%). Among enrollees with neither asthma nor PTSD, the adjusted risk ratio (aRR) for persistent GERS was elevated among: workers arriving at the WTC pile on $9 / 11$ (aRR $=1.6$; 95\% confidence interval $(\mathrm{Cl}) 1.3-2.1$ ) or working at the WTC site $>90$ days $(a R R=1.6 ; 1.4-2.0)$; residents exposed to the intense dust cloud on $9 / 11$ (aRR $=1.5 ; 1.0-2.3$ ), or who did not evacuate their homes (aRR =1.7; $1.2-2.3$ ); and area workers exposed to the intense dust cloud (aRR $=1.5 ; 1.2-1.8)$.

CONCLUSIONS: Disaster-related environmental exposures may contribute to the development of GERS. GERS may be accentuated in the presence of asthma or PTSD.

Am J Gastroenterol 2011; 106:1933-1941; doi:10.1038/ajg.2011.300; published online 6 September 2011

\section{INTRODUCTION}

Gastroesophageal reflux disease (GERD) is a chronic condition whose symptoms-heartburn and reflux-affect one-fifth of the population in a given week $(1,2)$. Long-standing symptoms of reflux can progress to Barrett's esophagus and esophageal cancer (3-5). GERD often coexists with asthma, posttraumatic stress disorder (PTSD), or other psychological comorbidities (6-8), although it is unclear which of these conditions may cause the others, or whether they are caused by common stressors (9-11). All three conditions
(GERD, asthma, PTSD) were found to be elevated in persons exposed to the World Trade Center (WTC) terrorist attacks of September 11 2001, in several populations with differing experiences (12-17). There is considerable evidence that asthma and PTSD were caused by WTC exposures $(13,15,16,18-20)$, but an independent effect of exposure on GERD has not been established.

In this report we examine associations between 9/11 exposures and subsequent development of symptoms indicative of GERD (which we denote as gastroesophageal reflux symptoms (GERS))

${ }^{1}$ New York City Department of Health and Mental Hygiene, New York, New York, USA; ${ }^{2}$ Department of Epidemiology, Mailman School of Public Health, Columbia University, New York, New York, USA; ${ }^{3}$ Epidemiology and Biostatistics Program, City University of New York School of Public Health-Hunter College, New York, New York, USA. Correspondence: Jiehui Li, MBBS, MSc, New York City Department of Health and Mental Hygiene, 42-09 28th Street, Long Island City, New York, 11101, USA. E-mail: jli3@health.nyc.gov

Received 4 April 2011; accepted 10 August 2011 
in a World Trade Center Health Registry ("Registry") cohort. We evaluated these associations separately in persons with and without comorbid post-9/11 asthma or PTSD in order to determine whether exposure predicts excess GERS independently of asthma or PTSD. We further describe the cumulative incidence of GERS in persons with varying degrees of comorbidity in order to better appreciate the unrecognized magnitude of the problem and to underscore the necessity for coordinated medical care for persons with physical and mental health problems related to the WTC attacks.

\section{METHODS}

\section{Study enrollees}

The Registry was established in 2002 to monitor the health consequences of exposure to the attacks on the WTC towers and the aftermath. In total, 71,437 persons voluntarily enrolled and completed an initial interview in 2003-04 (Wave 1, W1) (21). A follow-up questionnaire (Wave 2, W2) was completed in $2006-07$ by $68 \%$ of the $\mathrm{W} 1$ adult enrollees (16).

A total of 37,118 adult enrollees who completed the W2 survey were included in this analysis. We excluded: (i) those who reported having GERD symptoms before September $112001(n=5,735)$ or who had incomplete or missing information on GERD symptoms at W1 ( $n=356)$; (ii) 2,713 enrollees who were employed by the Fire Department of New York City, because periodic physical examinations required for continued employment as firefighters may introduce substantial surveillance bias; (iii) students and school staff $(n=74)$, because of small numbers; (iv) those who reported esophageal or stomach cancer $(n=10)$ at $\mathrm{W} 1$, or were pregnant on 9/11 $(n=316)$.

Eligibility groups were categorized in a mutually exclusive, hierarchical order, with rescue/recovery workers and volunteers $(n=16,008)$ first, followed by lower Manhattan residents $(n=5,642)$, lower Manhattan area workers $(n=13,491)$, and passersby, south of Chambers Street in lower Manhattan on 9/11 $(n=1,977)(16)$.

\section{Outcome}

Both W1 and W2 questionnaires elicited a history of heartburn, indigestion, or reflux symptoms (GERS) suggestive of possible GERD. At W1, we asked, "Since 9/11, have you experienced heartburn, indigestion, or reflux?" If the answer was positive, we further asked, "Before 9/11 did you have heartburn, indigestion, or reflux?" At W2, we asked, "Have you experienced heartburn, indigestion, or reflux in the last 30 days?" and "Number of days" having the symptom. We categorized the study outcomes as post-9/11 GERS and persistent GERS. Post-9/11 GERS was defined as any new onset of GERS since September 112001 that was reported at W1. Although the W2 survey did not indicate whether enrollees experienced episodic GERS between W1 and W2, GERD symptoms are often intermittently present for more than 5 years (2). Therefore, we defined "persistent post-9/11 GERS" as reporting "post-9/11 GERS" at W1 and in the 30 days before W2.

\section{Exposure and covariates}

Disaster-related exposures were defined by experiences common to all groups as well as unique group-specific exposures as described in earlier Registry reports $(16,21)$. Common exposures included level of exposure to the dust/debris cloud on 9/11 from the collapse of the WTC towers (none, some, intense) and the number of specific traumatic events witnessed on $9 / 11$ $(0,1$ to $2, \geq 3)$ (16). Group-specific exposures to 9/11-related dust/debris included the date of arrival and days worked at WTC site (for rescue workers), absence of evacuation from and damage to home (for residents), and damage to workplace (for area workers).

Covariates included demographics (age at enrollment, sex, race/ethnicity, household income in 2002, and education), smoking status, and source of enrollment (self- vs. list-identification) obtained in W1. List-identification was a method of recruitment where potential enrollees were contacted for interviews from the lists provided by the employers, government agencies, and other entities. Self-identified enrollees either pre-registered on the Registry website and were contacted for an interview or called a toll-free number for an interview.

Enrollees were classified as having asthma, based on selfreported history of physician-diagnosed asthma or reactive airways dysfunction syndrome. They were classified as having PTSD, based on self-reported history of PTSD diagnosis or responses to the PTSD Checklist-Civilian Version, a validated, self-reported 17-item symptom scale that corresponds to DSM-IV diagnostic criteria and which is often used when a clinical interview is not feasible $(22,23)$. A score of $\geq 44$ was indicative of probable PTSD. Using these definitions, comorbidity in this report refers to those enrollees with asthma, PTSD, or both.

\section{Data analysis}

We calculated the cumulative incidence of post-9/11 GERS by dividing the number of persons with post-9/11 GERS during the study period by the number of subjects at-risk for post-9/11 GERS at the beginning of the study. We describe the distributions of post-9/11 GERS and persistent GERS by demographics, enrollment source, smoking status, and each eligibility group according to the 9/11 exposure variables and the presence or absence of asthma and PTSD. The Cochran-Armitage trend test (two-sided) (24) was used to determine whether the gradients of exposure were associated with outcomes.

The cumulative incidence ratio (risk ratio (RR)) for persistent GERS in relation to 9/11-related exposures was analyzed using log-binomial regression $(25,26)$, with separate models for each eligibility group, and adjustment for age, sex, race/ethnicity, enrollment source, and smoking status. Log-binomial regression was used because the outcome prevalence exceeds $10 \%$ in our sample and this method enables us to compute the RR directly. Education and income were not included in the multivariate models to preserve the statistical efficiency because the likelihood ratio test for the difference between models with and without these two variables provided a statistically nonsignificant value. To study the potential effects of asthma and PTSD, the associations of GERS with 9/11-related exposures were examined in the bivariate and multivariate analyses separately for the comorbid and non-comorbid groups. 
All data analyses were performed using the SAS software (SAS Institute, Cary, NC, version 9.1.3). This study was approved by the Institutional Review Boards of the NYC Department of Health and Mental Hygiene, and the Centers for Disease Control and Prevention.

\section{RESULTS}

The overall cumulative incidence of post-9/11 GERS was $20.3 \%$ $(n=7,536)$ at $\mathrm{W} 1 ; 64.6 \%(n=4,870)$ of those affected were deemed to have persistent GERS at W2 (cumulative incidence of $13.1 \%$ ). The distributions of these two outcomes by subgroup are shown in Table 1. Post-9/11 GERS was reported equally by men and women, and less often among persons $18-24$ years of age (10.9\%). It was reported more frequently among the Hispanics (31.6\%), adults with income below $\$ 25,000$ (25.6\%), current smokers (26.3\%), and persons with lower education levels (e.g., 30.4\% among those without a high school diploma). Among the four eligibility groups, post-9/11 GERS was highest in rescue workers (22.5\%) and lowest in residents $(16.0 \%)$.

The cumulative incidence of GERS by 9/11-related exposures within each eligibility group is shown in Table 2 . Across all the eligibility groups, higher cumulative incidences of both post-9/11 GERS and persistent GERS were found among those exposed to the intense dust cloud and those who witnessed 3 or more traumatic events on 9/11. These exposures were common to all groups. Exposure-GERS relationships specific to particular eligibility groups were also observed. The highest cumulative incidence rates of post-9/11 GERS and persistent GERS were found among rescue/recovery workers who arrived at the WTC pile on $9 / 11$ (33.5\% post-9/11 GERS, $25.4 \%$ persistent GERS) or worked $>90$ days at the WTC site $(30.8 \%$ post-9/11 GERS, $21.8 \%$ persistent GERS), and among residents (23.1\% post-9/11 GERS, $14.6 \%$ persistent GERS) and area workers (26.6\% post-9/11 GERS, $17.9 \%$ persistent GERS) who returned to damaged homes or workplaces, respectively, covered with a heavy layer of dust.

The cumulative incidence of post-9/11 GERS increased with the number of comorbid conditions (i.e., asthma and PTSD), from $13.7 \%$ among those with neither condition to $45.8 \%$ among those with both conditions. The cumulative incidence of persistent GERS also increased substantially in the presence of asthma (12.9\%, $n=670)$, PTSD $(24.4 \%, n=1,521)$, and both asthma and PTSD $(35.5 \%, n=902)$, compared with adults with neither condition $(7.7 \%, n=1,777)$. Figures 1 and 2 illustrate the incidence of persistent GERS in relation to key 9/11 exposure variables, within four comorbid strata for both rescue/recovery workers (Figure 1) and non-rescue workers (Figure 2). Strong dose-response gradients are apparent in both groups, and are somewhat steeper among rescue/recovery workers.

Table 3 presents crude and adjusted RRs for persistent GERS for each eligibility group, in those with and without comorbid conditions (history of asthma and/or PTSD), respectively.

\section{Rescue/recovery workers}

Earlier arrival and longer duration of work at the WTC site were significantly associated with persistent GERS in bivariate and mul- tivariate analyses; the highest risks were observed in those who arrived on $9 / 11$ on the pile and stayed at the site for the longest time ( $>90$ days). The aRRs were greater among the non-comorbid group (arrival on $9 / 11$ aRR $=1.6 ; 95 \%$ confidence interval $(\mathrm{CI})=$ $1.3-2.1$; $>90$ days worked at $\mathrm{WTC}$ aRR $=1.6,95 \% \mathrm{CI}=1.4-2.0$ ) than among the comorbid group (arrival on $9 / 11 \mathrm{aRR}=1.3 ; 95 \%$ $\mathrm{CI}=1.1-1.6 ;>90$ days worked at WTC aRR $=1.4,95 \% \mathrm{CI}=$ 1.2-1.5). Risk ratios were attenuated after multivariate adjustment, but remained statistically significant in both the non-comorbid and comorbid groups, and continued to display exposure gradients. Exposure to dust cloud became nonsignificant in both the noncomorbid and comorbid rescue workers after adjustment.

\section{Residents}

Among non-comorbid residents, having been caught in the dust cloud was significantly associated with persistent GERS at the "some" $(\mathrm{aRR}=1.5,95 \% \mathrm{CI}=1.03-2.2)$ and "intense" dust cloud exposure levels ( $\mathrm{aRR}=1.5,95 \% \mathrm{CI}=1.0-2.3)$ (Table 3). Non-comorbid residents who did not evacuate their homes were more likely to have persistent GERS than those who did evacuate $(\mathrm{aRR}=1.7,95 \%$ $\mathrm{CI}=1.2-2.3$ ). However, exposure to homes damaged with a heavy layer of dust was not associated with persistent GERS among these residents, after adjustment.

Among comorbid residents, significantly elevated RRs were seen for those whose homes were damaged with a heavy layer of dust $(\mathrm{aRR}=1.3,95 \% \mathrm{CI}=1.0-1.6)$. On the other hand, being caught in the dust cloud, and absence of evacuation from home were not associated with persistent GERS after adjustment for covariates.

\section{Area workers}

Regardless of comorbidity, compared with the crude RR, the risk of persistent GERS among those with intense dust cloud exposure was attenuated but remained significant after adjustment. Similar to residents, the aRR for damage to the workplace without a heavy layer of dust among area workers was statistically significant for persistent GERS in the non-comorbid group $(\mathrm{aRR}=1.3,95 \%$ $\mathrm{CI}=1.02-1.6$ ), but the aRR for damage to workplace with a heavy layer of dust was not statistically significant for persistent GERS in the comorbid group $(\mathrm{aRR}=1.2,95 \% \mathrm{CI}=0.99-1.3)$.

\section{Passersby}

Intense dust cloud exposure was significantly associated with persistent GERS among the comorbid passersby (aRR $=1.7,95 \%$ $\mathrm{CI}=1.2-2.6$ ), but not among the non-comorbid passersby after adjustment for covariates.

\section{DISCUSSION}

There were three main findings in this study. First, cumulative incidence of post-9/11 persistent GERS was positively associated with higher intensity 9/11-related exposures compared with lower exposures, and this finding was consistent across the rescue/ recovery workers, local residents, and area workers. Second, post-9/11 persistent GERS was independently associated with 9/11-related exposures when persons with asthma or PTSD were 
Table 1. Cumulative incidence of post-9/11 GERS and persistent GERS among adult enrollees who participated in Wave 1 and Wave 2 surveys $(N=37,118)$

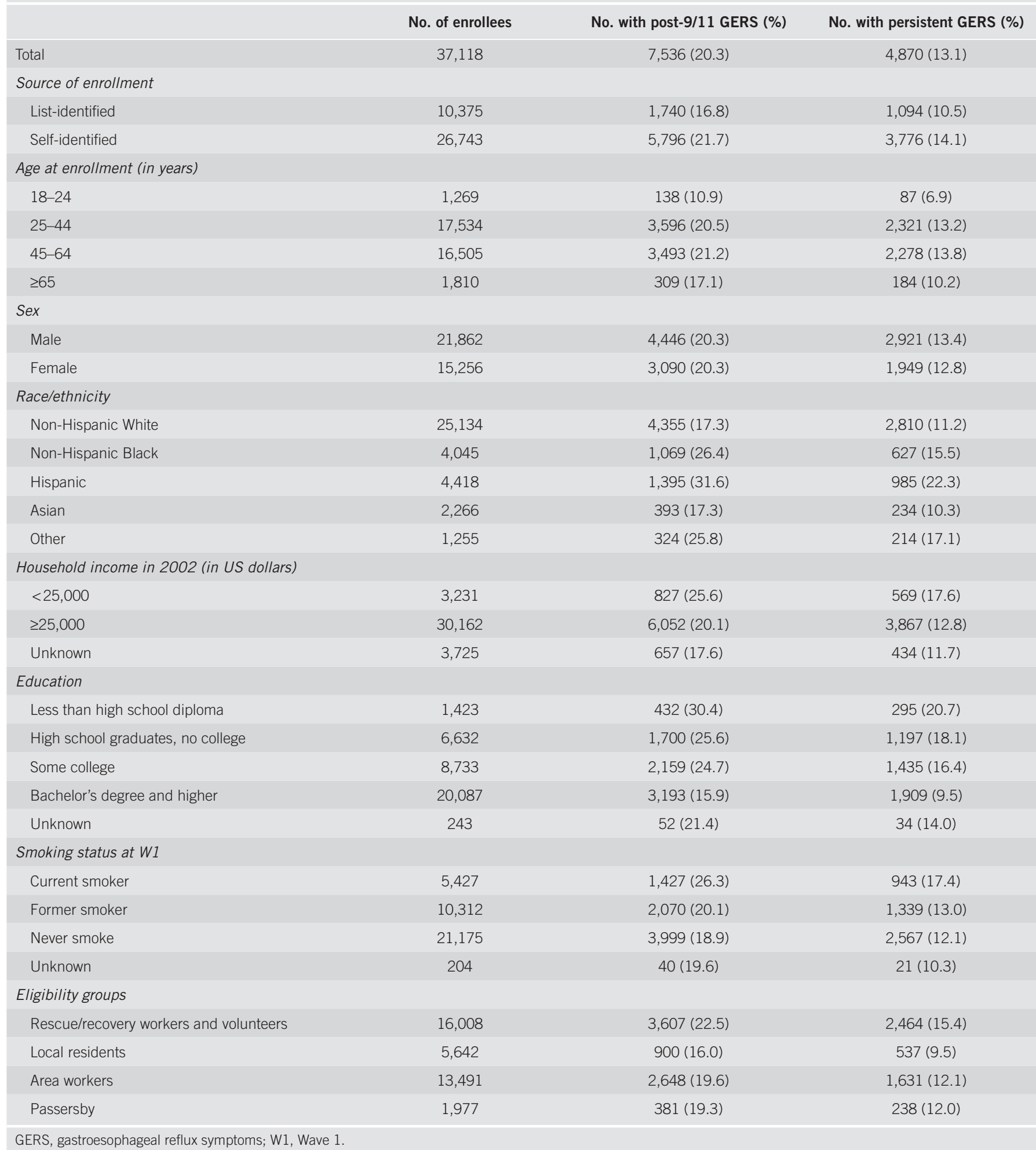

excluded. Third, those highly exposed in non-comorbid group were more likely to have persistent GERS than those in comorbid group.
The cumulative incidence of post-9/11 GERS in our study population was 20.3\% between September 112001 and the initial interview held in $2003-04$. GERS persisted for $64.6 \%$ of those affected 
Table 2. Post-9/11 GERS and persistent GERS by eligibility group and 9/11-related exposures among adult enrollees who participated in both Wave 1 and Wave 2 surveys $(N=37,118)^{a}$

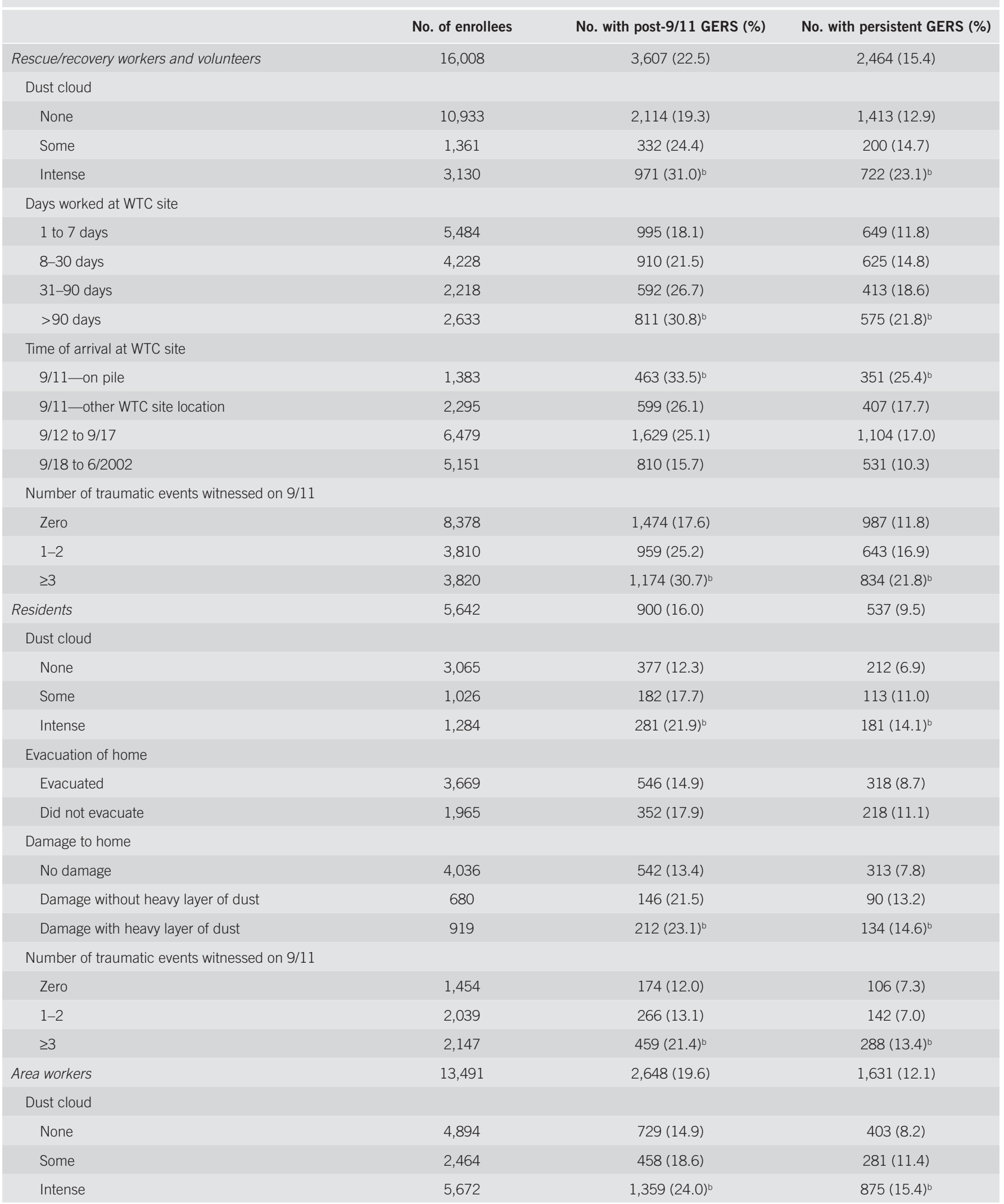


Table 2. Continued

No. of enrollees

10,093

2,000

1,384

Damage with heavy layer of dust

Number of traumatic events witnessed on 9/11

Zero

1-2

$\geq 3$

Passersby

Dust cloud

None

Some

Intense

Number of traumatic events witnessed on 9/11

Zero

$1-2$

$\geq 3$
1,125

5,615

6,751

1,977

814

414

684

154

800

1,023
No. with post-9/11 GERS (\%)

No. with persistent GERS (\%)

$1,812(18.0)$

$1,088(10.8)$

464 (23.2)

294 (14.7)

$368(26.6)^{\mathrm{b}}$

$247(17.9)^{\mathrm{b}}$

$161(14.3)$

89 (7.9)

933 (16.6)

552 (9.8)

$1,554(23.0)^{b}$

$990(14.7)^{\mathrm{b}}$

381 (19.3)

238 (12.0)

117 (14.4)

69 (8.5)

66 (15.9)

38 (9.2)

$186(27.2)^{b}$

$127(18.6)^{b}$

27 (17.5)

18 (11.7)

125 (15.6)

75 (9.4)

$229(22.4)^{\mathrm{b}}$

GERS, gastroesophageal reflux symptoms; WTC, World Trade Center.

aThe sum under each variable may not be equal to the total number, because enrollees with unknown information were not presented in this Table.

${ }^{b}$ Cochran-Armitage test for trend, $P<0.01$.

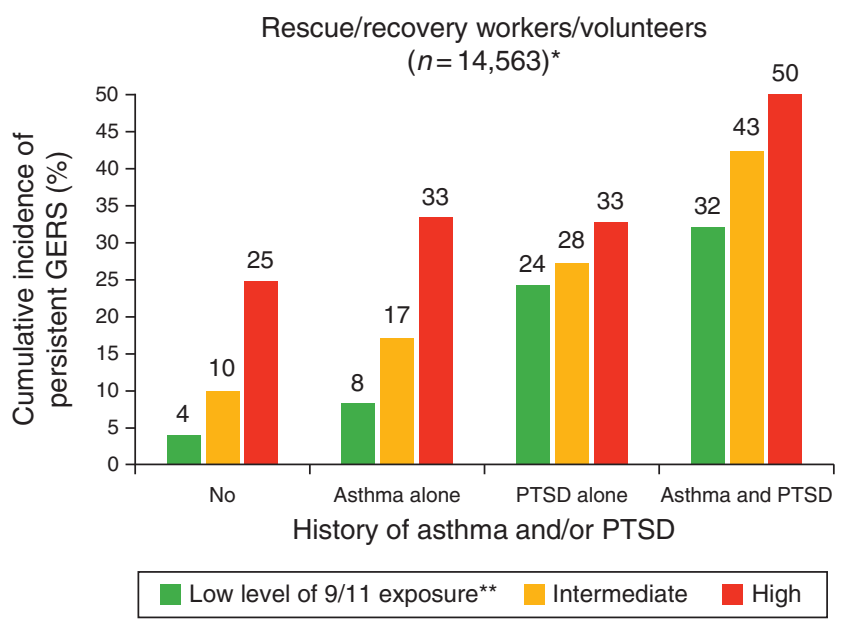

Figure 1. Cumulative incidence of persistent gastroesophageal reflux symptoms (GERS) by 9/11-related exposure and comorbid condition among rescue/recovery workers who participated in both Wave 1 and Wave 2 surveys. *Excluding those with unknown information on date of arrival and days worked at the World Trade Center (WTC) site. ** Low level of 9/11-related exposure $=$ began work after 17 September 2001 and stayed for 1-7 days at the WTC site; High=worked on the pile on 11 September 2001 for >90 days; Intermediate $=$ refers to all exposures that are by definition greater than low and less than high exposure level. PTSD, posttraumatic stress disorder.

up to at least 6 years after 9/11. Our findings are consistent with those of a 9/11-related screening program (27) that reported an incidence of $18.9 \%$ for indigestion and heartburn symptoms 9-15
Residents, area workers, or passersby $(n=20,317)^{\star}$

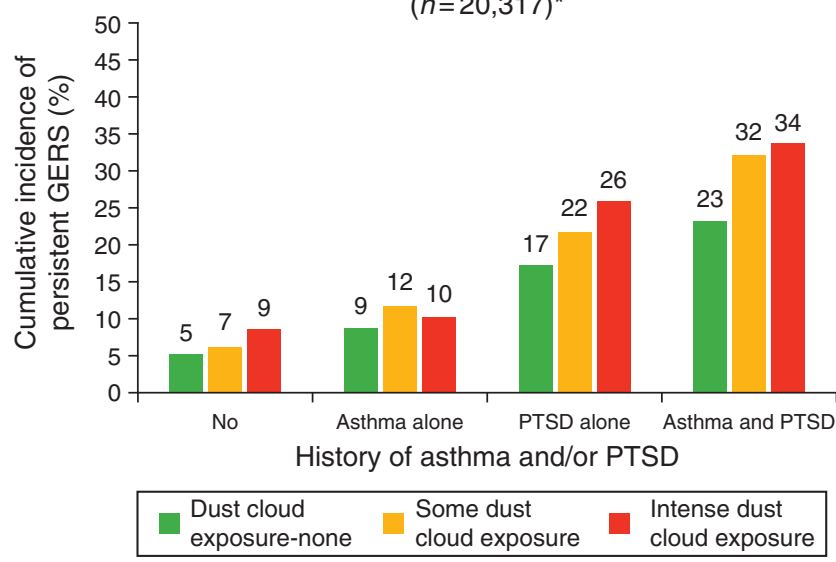

Figure 2. Cumulative incidence of persistent gastroesophageal reflux symptoms (GERS) by 9/11-related dust cloud exposure and comorbid condition among residents, area workers, and passersby who participated in both Wave 1 and Wave 2 surveys. * Excluding those with unknown information on dust cloud exposure $(N=793)$. PTSD, posttraumatic stress disorder.

months after 9/11; symptoms were present among $80 \%$ in the month before screening.

Many of these common and group-specific exposures were previously found to be associated with post-9/11 new-onset asthma and PTSD $(16,19)$; hence it is not surprising that cumulative incidence of GERS is greater in persons with one or both of these conditions than in those with neither. An important question is whether 
GERS is independently associated with $9 / 11$ exposures as data in Table 3 suggest. The large cohort size permitted separate estimates of RRs for exposure-GERS associations in enrollees with and with- out comorbid asthma or PTSD. Most associations were statistically significant and tended to be stronger in the non-comorbid than in the comorbid groups, suggesting that the increased incidence of

Table 3. Bivariate and multivariate analyses of persistent GERS associated with 9/11-related risk factors by eligibility groups and comorbid conditions

\begin{tabular}{|c|c|c|c|c|}
\hline \multirow[b]{2}{*}{ Variable } & \multicolumn{2}{|c|}{ Non-comorbid group $(N=23,153)^{a}$} & \multicolumn{2}{|c|}{ Comorbid group $(N=13,965)^{\mathrm{a}}$} \\
\hline & $\begin{array}{l}\text { Crude risk ratio }(95 \% \\
\text { confidence interval }(\mathrm{CI}))\end{array}$ & $\begin{array}{c}\text { Adjusted RR } \\
(95 \% \mathrm{Cl})^{\mathrm{b}}\end{array}$ & $\begin{array}{l}\text { Crude RR } \\
(95 \% \mathrm{Cl})\end{array}$ & $\begin{array}{l}\text { Adjusted RR } \\
(95 \% \mathrm{CI})^{\mathrm{c}}\end{array}$ \\
\hline \multicolumn{5}{|c|}{ Dust cloud exposure on 9/11 (referent=none) } \\
\hline Some & $1.1(0.8-1.3)$ & $0.8(0.6-1.01)$ & $1.0(0.9-1.2)$ & $0.9(0.7-1.1)$ \\
\hline \multicolumn{5}{|c|}{ Days working at the WTC site (referent=1 to 7 ) } \\
\hline 8 to 30 & $1.3(1.1-1.5)$ & $1.2(1.04-1.5)$ & $1.3(1.1-1.4)$ & $1.2(1.1-1.4)$ \\
\hline 31 to 90 & $1.7(1.4-2.0)$ & $1.4(1.2-1.8)$ & $1.4(1.2-1.6)$ & $1.3(1.1-1.5)$ \\
\hline$>90$ & $1.9(1.5-2.2)$ & $1.6(1.4-2.0)$ & $1.6(1.4-1.8)$ & $1.4(1.2-1.5)$ \\
\hline \multicolumn{5}{|c|}{ Time of arrival at the WTC site (referent=9/18 to 6/2002) } \\
\hline $9 / 12$ to $9 / 17$ & $1.7(1.5-2.0)$ & $1.2(1.1-1.5)$ & $1.3(1.2-1.5)$ & $1.2(1.03-1.3)$ \\
\hline \multicolumn{5}{|l|}{ Local residents } \\
\hline \multicolumn{5}{|c|}{ Dust cloud exposure on 9/11 (referent=none) } \\
\hline Some & $1.6(1.1-2.3)$ & $1.5(1.03-2.2)$ & $1.4(1.1-1.9)$ & $1.2(0.9-1.5)$ \\
\hline Intense & $1.6(1.1-2.3)$ & $1.5(1.0-2.3)$ & $1.6(1.3-2.0)$ & $1.2(0.96-1.6)$ \\
\hline \multicolumn{5}{|l|}{ Evacuation of home (referent=evacuated) } \\
\hline Did not evacuate & $1.6(1.2-2.1)$ & $1.7(1.2-2.3)$ & $1.1(0.9-1.4)$ & $1.2(0.96-1.4)$ \\
\hline \multicolumn{5}{|l|}{ Damage to home (referent= no damage) } \\
\hline Damage without heavy layer of dust & $1.5(1.0-2.3)$ & $1.7(1.1-2.5)$ & $1.4(1.1-1.9)$ & $1.1(0.8-1.4)$ \\
\hline \multicolumn{5}{|c|}{ Dust cloud exposure on 9/11 (referent=none) } \\
\hline \multicolumn{5}{|l|}{ Damage to office (referent= no damage) } \\
\hline Damage without heavy layer of dust & $1.4(1.1-1.7)$ & $1.3(1.02-1.6)$ & $1.2(1.1-1.4)$ & $1.1(0.98-1.3)$ \\
\hline Damage with heavy layer of dust & $1.3(1.0-1.7)$ & $1.1(0.9-1.5)$ & $1.4(1.2-1.6)$ & $1.2(0.99-1.3)$ \\
\hline \multicolumn{5}{|l|}{ Passersby } \\
\hline \multicolumn{5}{|c|}{ Dust cloud exposure on 9/11 (referent=none) } \\
\hline Some & $1.0(0.5-1.7)$ & $1.0(0.5-1.8)$ & $1.1(0.7-1.9)$ & $0.9(0.5-1.5)$ \\
\hline Intense & $1.5(0.95-2.4)$ & $1.5(0.9-2.5)$ & $2.2(1.5-3.1)$ & $1.7(1.2-2.6)$ \\
\hline
\end{tabular}

GERS, gastroesophageal reflux symptoms; RR, risk ratio; WTC, World Trade Center.

aSample size varies with different models; enrollees with missing values were excluded from the analysis.

${ }^{b}$ Age at enrollment, gender, ethnicity, smoking status, source of enrollment, number of traumatic events witnessed on 9/11, and variables listed for the corresponding eligibility group in the table were included in the multivariate analysis.

'Age at enrollment, gender, ethnicity, smoking status, source of enrollment, number of traumatic events witnessed on 9/11, history of asthma and/or post-9/11 posttraumatic stress disorder (PTSD) and variables listed for the corresponding eligibility group in the table were included in the multivariate analysis. 
GERS among the exposed is not merely due to or modified by the co-occurrence of GERS with asthma or PTSD, but is independently related to exposures.

Several possible mechanisms may account for an independent association between 9/11 exposures and GERS. First, the ingestion of 9/11 environmental contaminants may have directly resulted in gastroesophageal injury. Ingestion of alkaline materials in the dust cloud (28) was a suggested cause of new or worsening GERD in WTC-exposed firefighters (29). Alkaline cement dust, one of the major constituents of WTC dust, has been associated in occupational studies with both adverse respiratory outcomes such as asthma $(30,31)$ and reflux-like dyspepsia (32), but the biological mechanism is not clear.

Even though persons who had exceeded the threshold for probable PTSD were excluded, subsyndromal PTSD and other psychological distress may have been a mediator in the non-comorbid group. In a sensitivity analysis, we found very little attenuation of the associations between 9/11 exposures and GERS when we also excluded persons who exceeded the threshold for moderate to serious psychological distress at either W1 or W2, as measured by the Kessler 6-item scale from the non-comborbid group (33) (data not shown). In-depth studies are required to further elucidate the relationship between GERS and 9/11 exposure.

We acknowledge some limitations in this study. First, use of GERS rather than clinically diagnosed GERD may have overestimated the disease in the study population. Because "indigestion", a less specific symptom of GERD than "heartburn or reflux", was asked along with "heartburn or reflux", and the questions regarding GERS we used in the surveys were not validated, we might have included non-GERS in the GERS group. However, in our subanalysis, nearly $80 \%$ of 4,870 enrollees who had persistent post-9/11 GERS reported at least 4 days of having heartburn, indigestion, or reflux, and 54\% reported at least 9 days of having heartburn, indigestion, or reflux in a 30-day period. The likelihood of including non-GERS persons may be minimal. Second, the non-persistent GERS group may contain those who had GERS before 9/11 but did not report any GERS in W1, leading to an underestimate of the cumulative incidence of persistent GERS. Third, information on known risk factors for GERS was unavailable, including obesity, family history, and use of medication $(34,35)$. Fourth, if enrollees with GERS or with higher levels of 9/11-related exposure were more likely to participate in W2, we might have overestimated GERS. However, the Registry adult enrollees who participated in W2 were not statistically different from those who participated only in $\mathrm{W} 1$, in terms of exposure to dust cloud, traumatic events, or injury as a result of 9/11 (16). In addition, among W2 participants, the percentage of post-9/11 GERS reported at W1 was virtually identical to that in W2 non-participants. Fifth, if rescue/ recovery workers who had ever received services from 9/11-related medical monitoring and treatment programs for first responders were more likely to report persistent GERS, potential detection bias may occur. But "receipt of 9/11-related medical services" did not confound the association of GERS with 9/11-exposures (data not shown). Finally, any possible tendency to over-report symptoms is unlikely to affect our findings because we used internal comparisons that would likely lead to a non-differential misclassification of GERS, reducing the observed relative risk toward the null value.

In summary, we found the incidence of post-9/11 GERS to be higher in individuals with asthma or PTSD than in those without either condition, and highest in those with both comorbid conditions. Although our data are not sufficiently sensitive to demonstrate conclusively the time sequence of these three conditions and hence unable to probe causal relationships, they are consistent with prior studies showing a higher likelihood for asthmatics to develop GERD than non-asthmatics (6), and with studies showing an increased propensity for patients with psychological symptoms also to report GERD symptoms (7). Post-9/11 or persistent GERS may pose a potential burden on healthcare resources and likely deepen the impairment of health-related quality of life for the WTC-exposed population that was already affected by other health effects arising from the 9/11 disaster. Clinicians need to be sensitive to the close association among the three conditions and the strong possibility that patients with 9/11 GERS may have comorbid respiratory or mental health problems. They should also be aware of published clinical guidelines, for treatment of persons exposed to the WTC disaster, which contain recommendations for coordinating physical and mental health treatment (36).

\section{ACKNOWLEDGMENTS}

We gratefully acknowledge the participation of all Registry enrollees. We thank our Community Advisory Board, Labor Advisory Committee, and the Scientific Advisory Committee for their helpful inputs. We also thank Dr. James Hadler, Dr. Carolyn Greene, and Dr. Hannah Jordan from the NYCDOHMH, and Dr. Dori B. Reissman from the National Institute for Occupational Safety and Health, for their critical and thorough review of the manuscript.

\section{DISCLAIMER}

The views expressed in this article are those of the authors and do not necessarily represent the official views of the $\mathrm{CDC} / \mathrm{NIOSH}$, ATSDR, CDC/NCEH, or NYCDOHMH.

\section{CONFLICT OF INTEREST}

Guarantor of the article: Jiehui Li, MBBS, MSc. Specific author contributions: Had full access to all of the data in the study and took responsibility for the integrity of the data and the accuracy of the data analysis: Li and Brackbill; conceived the study: LET; completed data analysis: JL and SM. All authors participated in the study design, data collection, data interpretation, and drafting of the manuscript.

Financial support: This study was supported by the Cooperative Agreement 1U50/OH009739 from the National Institute for Occupational Safety and Heath (NIOSH) and Cooperative Agreement U50/ATU272750 from the Agency for Toxic Substances and Disease Registry (ATSDR) (which included support from the National Center for Environmental Health (NCEH)) of the 
Centers for Disease Control and Prevention (CDC), and by the New York City Department of Health and Mental Hygiene (NYCDOHMH).

Role of the Sponsors: The NIOSH provided review on the manuscript. ATSDR provided review and clearance.

Potential competing interests: None.

\section{Study Highlights}

\section{WHAT IS CURRENT KNOWLEDGE}

Excess gastroesophageal reflux disease was reported in several populations exposed to the September 112001 (9/11) terrorist attacks on the World Trade Center (WTC), and often coexists with asthma, posttraumatic stress disorder (PTSD), or other psychological comorbidities.

There is considerable evidence that asthma and PTSD were caused by WTC exposures, but an independent effect of exposure on GERD has not been established.

\section{WHAT IS NEW HERE}

The increased incidence of GERS is independently related to exposure and not simply due to or modified by the co-occurrence of GERS with asthma or PTSD.

The cumulative incidence of persistent GERS increased with increasing 9/11 exposure; it was higher in those with comorbid asthma and PTSD than in those with neither comorbid condition.

\section{REFERENCES}

1. Dent J, El-Serag HB, Wallander MA et al. Epidemiology of gastrooesophageal reflux disease: a systematic review. Gut 2005;54:710-7.

2. Locke GR III, Talley NJ, Fett SL et al. Prevalence and clinical spectrum of gastroesophageal reflux: a population-based study in Olmsted County, Minnesota. Gastroenterology 1997;112:1448-56.

3. Lieberman DA, Oehlke M, Helfand M. Risk factors for Barrett's esophagus in community-based practice. GORGE consortium. Gastroenterology Outcomes Research Group in Endoscopy. Am J Gastroenterol 1997;92: 1293-7.

4. Lagergren J, Bergström R, Lindgren A et al. Symptomatic gastroesophageal reflux as a risk factor for esophageal adenocarcinoma. $\mathrm{N}$ Engl J Med 1999;340:825-31.

5. Shaheen N, Ransohoff DF. Gastroesophageal reflux, Barrett esophagus, and esophageal cancer: scientific review. JAMA 2002;287:1972-81.

6. Havemann BD, Henderson CA, El-Serag HB. The association between gastro-oesophageal reflux disease and asthma: a systematic review. Gut 2007;56:1654-64.

7. Mizyed I, Fass SS, Fass R. Review article: gastro-oesophageal reflux disease and psychological comorbidity. Aliment Pharmacol Ther 2009;29:351-8.

8. Avidan B, Sonnenberg A, Giblovich $\mathrm{H}$ et al. Reflux symptoms are associated with psychiatric disease. Aliment Pharmacol Ther 2001;15:1907-12.

9. Wright RJ, Rodriguez M, Cohen S. Review of psychosocial stress and asthma: an integrated biopsychosocial approach. Thorax 1998;53:1066-74.

10. Mayer EA. The neurobiology of stress and gastrointestinal disease. Gut 2000;47:861-9.

11. Ruigómez A, Rodríguez LA, Wallander MA et al. Gastroesophageal reflux disease and asthma: a longitudinal study in UK general practice. Chest 2005;128:85-93.

12. Webber MP, Gustave J, Lee R et al. Trends in respiratory symptoms of firefighters exposed to the World Trade Center disaster: 2001-2005. Environ Health Perspect 2009;117:975-80.
13. de la Hoz RE, Shohet MR, Chasan R et al. Occupational toxicant inhalation injury: the World Trade Center (WTC) experience. Int Arch Occup Environ Health 2008;81:479-85.

14. Brackbill RM, Thorpe LE, DiGrande L et al. Surveillance for World Trade Center disaster health effects among survivors of collapsed and damaged buildings. MMWR Surveill Summ 2006;55:1-18.

15. Perrin MA, DiGrande L, Wheeler K et al. Differences in PTSD prevalence and associated risk factors among World Trade Center disaster rescue and recovery workers. Am J Psychiatry 2007;164:1385-94.

16. Brackbill RM, Hadler JL, DiGrande L et al. Asthma and posttraumatic stress symptoms 5 to 6 years following exposure to the World Trade Center terrorist attack. JAMA 2009;302:502-16.

17. DiGrande L, Perrin MA, Thorpe LE et al. Posttraumatic stress symptoms, PTSD, and risk factors among lower Manhattan residents 2-3 years after the September 11, 2001 terrorist attacks. J Trauma Stress 2008;21:264-73.

18. Galea S, Ahern J, Resnick H et al. Psychological sequelae of the September 11 terrorist attacks in New York City. N Engl J Med 2002;346:982-7.

19. Wheeler K, McKelvey W, Thorpe L et al. Asthma diagnosed after 11 September 2001 among rescue and recovery workers: findings from the World Trade Center Health Registry. Environ Health Perspect 2007;115:1584-90.

20. Stellman JM, Smith RP, Katz CL et al. Enduring mental health morbidity and social function impairment in world trade center rescue, recovery, and cleanup workers: the psychological dimension of an environmental health disaster. Environ Health Perspect 2008;116:1248-53.

21. Farfel M, DiGrande L, Brackbill R et al. An Overview of 9/11 Experiences and Respiratory and Mental Health Conditions among World Trade Center Health Registry Enrollees. J Urban Health 2008;85:880-909.

22. Blanchard EB, Jones-Alexander J, Buckley TC et al. Psychometric properties of the PTSD Checklist (PCL). Behav Res Ther 1996;34:669-73.

23. Ruggiero KJ, Del Ben K, Scotti JR et al. Psychometric properties of the PTSD checklist-Civilian Version. J Trauma Stress 2003;16:495-502.

24. Agresti A. Categorical Data Analysis, 2nd Ed John Wiley \& Sons: New York, 2002.

25. Greenland S. Model-based estimation of relative risks and other epidemiologic measures in studies of common outcomes and in case-control studies. Am J Epidemiol 2004;160:301-5.

26. Spiegelman D, Hertzmark E. Easy SAS calculations for risk or prevalence ratios and differences. Am J Epidemiol 2005;162:199-200.

27. Centers for Disease Control and Prevention. Physical Health Status of World Trade Center Rescue and Recovery Workers and Volunteers New York City, July 2002-August 2004. MMWR Morb Mortal Wkly Rep 2004;53:807-17.

28. Lioy PJ, Georgopoulos P. The anatomy of the exposures that occurred around the World Trade Center site: 9/11 and beyond. Ann NY Acad Sci 2006;1076:54-79.

29. Prezant DJ, Weiden M, Banauch GI et al. Cough and bronchial responsiveness in firefighters at the World Trade Center site. N Engl J Med 2002;347:806-15.

30. Al-Neaimi YI, Gomes J, Lloyd OL. Respiratory illnesses and ventilatory function among workers at a cement factory in a rapidly developing country. Occup Med (Lond) 2001;51:367-73.

31. Maciejewska AG, Bielichowska-Cybula G. Biological effect of cement dust. Med Pr 1991;42:281-9.

32. Coppeta L, Pietroiusti A, Magrini A et al. Prevalence and characteristics of functional dyspepsia among workers exposed to cement dust. Scand J Work Environ Health 2008;34:396-402.

33. Kessler RC, Andrews G, Colpe LJ et al. A short screening scales to monitor population prevalence and trends in nonspecific psychological distress. Psychol Med 2002;32:959-76.

34. Locke GR III, Talley NJ, Fett SL et al. Risk factors associated with symptoms of gastroesophageal reflux. Am J Med 1999;106:642-9.

35. Ruhl CE, Everhart JE. Overweight, but not high dietary fat intake, increases risk of gastroesophageal reflux disease hospitalization: the NHANES I Epidemiologic Follow-up Study. First National Health and Nutrition Examination Survey. Ann Epidemiol 1999;9:424-35.

36. Friedman S, Cone J, Eros-Sarnyai M et al. Clinical guidelines for adults exposed to the World Trade Center disaster. City Health Information 2008;27:41-54. 